\title{
Long-term programming of enhanced aggression by peripuberty stress in female rats
}

\author{
M. Isabel Cordero ${ }^{a, b, *}$, François Ansermet $^{b}$, Carmen Sandi $^{a}$
}

\author{
a Laboratory of Behavioral Genetics, Brain Mind Institute, School of Life Sciences, Ecole Polytechnique \\ Federale de Lausanne (EPFL), Lausanne, Switzerland \\ ${ }^{\mathrm{b}}$ Child and Adolescent Service of Psychiatry (SPEA), Hospital University of Geneva, Geneva, Switzerland
}

Received 4 March 2013; received in revised form 22 June 2013; accepted 10 July 2013

\section{KEYWORDS \\ Female aggression; Maternal behavior; Stress reactivity; Puberty; \\ Violence; Oxytocin; Vasopressin; Corticosterone; Long-term effects; Cohabitation female-male}

\begin{abstract}
Summary Human literature has linked adverse early life experiences with an increased risk to develop violent behaviors in both boys and girls. We have previously shown that male rats submitted to stress during the peripuberty period display as adults abnormal aggressive behavior against both male intruders and female partners. In the present study, we examined whether the same stress protocol would affect the development of aggressive behaviors in female rats. We evaluated the behavior of these peripuberty stressed female rats when confronted, at adulthood, with either female or male intruders, and during their cohabitation with male partners. Given that estrus cycle influences mood and aggressive behaviors, female aggressive behavior was assessed at different estrus cycle phases: estrus and diestrus, and during pregnancy and lactancy. Additionally, we evaluated postpartum plasma levels of vasopressin, oxytocin and corticosterone, hormones associated with aggression and the regulation of social behavior. Compared to control females, females submitted to stressful events during puberty exhibited higher and more sustained rates of aggression during adulthood independently on the estrus cycle or the sex of the intruder, and they had higher levels of plasma vasopressin. Significant correlations between plasma levels of vasopressin and corticosterone and aggressive behavior were also found. Strikingly, our results showed opposite intragroup correlations suggesting a different role of these hormones on aggression depending on life experiences. We provide here an animal model, devoid of cultural influences strongly supporting a role for biological factors in the development of aggressive behaviors following exposure to stressful events at puberty in females.
\end{abstract}

(C) 2013 Elsevier Ltd. All rights reserved.

\footnotetext{
* Corresponding author at: Ecole Polytechnique Fédérale de Lausanne, Brain Mind Institute, Laboratory of Behavioral Genetics, EPFL SV BMI LGC, AAB201 (Batiment AAB), Station 19, CH-1015 Lausanne, Switzerland. Tel.: +41 021693 1661; fax: +410216931660.

E-mail addresses: isabel.cordero@epfl.ch, Marialsabel.CorderoCampana@hcuge.ch (M.I. Cordero).
} 


\section{Introduction}

Puberty is a critical developmental period characterized by increased levels of gonadal steroid hormones with brain organizational influences, and increasing connectivity in social- and emotion-related areas (Blakemore, 2012). Many psychiatric disorders such as personality and mood disorders, addiction and schizophrenia start during adolescence, suggesting that the neurobiological events involved in remodeling neural circuits that occur during this period render the adolescent developing brain highly vulnerable to experiential input (Paus et al., 2008). Importantly, exposure to high rates of stress around puberty has been related to an increase of violent behaviors and personality disorders (PD) [note that a very high percentage of individuals that commit violent repeated offenses suffer PD (Putkonen et al., 2003)]; although this is typically reported in men (Wolff and Shi, 2012) evidence is also available in women (Foy et al., 2012). Animal research has shown that exposure to stressful events during puberty increases agonistic behaviors during adolescence and adulthood in several species (Delville et al., 2003; Sachser, 1993).

Since the physiological systems that sustain aggression (Albert and Walsh, 1984; Umukoro et al., 2012) and stress (de Kloet, 2010) are highly preserved throughout evolution, several animal models have been proposed to study the development of human aggressive behavior using rodents. However, very few models have included non-lactating females, possibly because aggressive behavior in female rodents is considered in general rare unless the females are protecting their offspring (for review, see Umukoro et al., 2012). Although the patterns of aggressive behavior differ between males and females (Blanchard et al., 1980), female rodents display non-reproduction related aggressive behavior as well (DeBold and Miczek, 1984). Furthermore, it is known that certain conditions and treatments increase aggressive behavior in both sexes, such as for example, electrical stimulation in the hypothalamus (Kruk et al., 1984), ethanol administration (Blanchard et al., 1987), or isolation (Malick, 1979; More, 2008). Differences between the degree to which male and female rodents react to environmental experiences affecting aggressive behavior have been reported as well. For instance, in rats, aggressive behavior measured after exposure to a single or repeated immobilization stress was found to be increased in females (Albonetti and Farabollini, 1993b) and decreased in males (Albonetti and Farabollini, 1993a); while exposure to maternal separation in early life led to increased aggression at adulthood in males (Veenema et al., 2006) and reduced aggression in females (Boccia and Pedersen, 2001). Therefore, these findings prevent the generalization of the effects of stress on aggressive behavior from males to females and highlight the need to investigate them in animals of both sexes.

We have previously shown that male rats submitted to stress - consisting of fear-inducing experiences - during the peripuberty period display as adults abnormal aggressive behavior against both male intruders (Marquez et al., 2013) and female partners (Cordero et al., 2012). In the present study, we examined whether the same stress protocol would affect the development of aggressive behaviors in female rats exposed to stress experiences during puberty. We evaluated the behavior of these peripuberty stressed female rats when confronted, at adulthood, with either female or male intruders, and during their cohabitation with male partners. Given that, in females, fluctuant levels of ovarian hormones related to the estrus cycle influence mood and aggressive behaviors (Lovick et al., 2005); female rats were submitted twice during adulthood to the resident intruder test, first in diestrus and the second exposure during estrus. Different protocols have been used to induce maternal aggression in female rats, including the exposure to an unfamiliar female (Bosch et al., 2010) or to a sexually naïve male intruder (Parmigiani et al., 1988; Johns et al., 1998). As the latter has been reported to induce more severe aggression and in order to assess inter-sexual aggressive behavior with low influence of mating and sexual behavior, maternal aggression against a male intruder was performed on postpartum day 7. Further, we evaluated plasma corticosterone levels, given the link between glucocorticoids and aggression (Haller et al., 2004) and the neuropeptides arginine vasopressin (AVP) and oxytocin (OXT) given their role in the regulation of social behavior and, particularly, aggression and maternal behaviors (Bosch and Neumann, 2012).

\section{Methods and materials}

\subsection{Animals}

The female experimental subjects were the offspring (F0) of Wistar Han rats (12 females and 12 males) purchased from Charles River Laboratories (Lyon, France) that were bred in our animal house. Taking into account that human studies have often found a high prevalence of psychopathology among first-degree relatives, in order to study the potential role of the stress in exacerbating pre-existent aggressive behavior, sisters were submitted to a different experimental condition, control or stress. In this way, at weaning, from each litter (with the exception of one litter that only had one female offspring) a pair of females of equivalent weight were separated and placed in a new home-cage together with two more females of equivalent weight from two other different litters ( 3 females per cage, no siblings between them). Efforts were made to ensure that each couple of cages was equally matched with three other females from the same three different litters. From these matched cages, all the animals from one of the cages were submitted to the same control or stress condition, while females from the other matched cage were submitted to the other experimental condition [ $N=12$ females per group; matched siblings between the groups: 11 pairs; note that as one female had no siblings, in order to maintain similar housing conditions for all the animals (namely three females per cage) one female from another litter had to be added to one cage, and therefore one pair of females (one in the control group and one in the stress group) were not siblings between them].

The male subjects (used either in the maternal aggression tests or as partners) were naive Wistar rats acquired at 10 week-old from Charles River Laboratories. Ovariectomized (OVX) females 3-month old, used as intruders in the residentintruder test, were obtained from Charles River. Ovariectomies were performed by the supplier (Charles River Laboratories) one week before shipment. Animals were maintained 
under controlled conditions ( $12 \mathrm{~h}$ light/dark cycle; lights on at $0700 \mathrm{~h} ; 22 \pm 2{ }^{\circ} \mathrm{C}$ ). Food and water were available ad libitum. With the exception of animals submitted to peripubertal stress and males used as intruders during the maternal aggression test (see procedure below), all animals were at least 3 months old at the beginning of the experiments. Except for the home-cage interactions and resident-intruder tests (see details below) and maternal care behavior observations (see details in supplementary material), animal testing occurred during the first half of the animals' light phase. All conducted procedures conformed to Swiss National Institutional Guidelines on Animal Experimentation and were approved through a license by the Swiss Cantonal Veterinary Office Committee for Animal Experimentation. All efforts were made to minimize animal suffering and to reduce the number of animals used.

\subsection{General experimental procedure}

The overall goal of the study was to evaluate the long-term effects of peripuberty stress on female aggressive behaviors during different behavioral tests and hormonal levels during the post-partum period (Fig. 1). On the last day of the stress period, blood samples were drawn on the same group of stress animals and a subset of control females only exposed to the elevated platform on p42 (see details below) to assess plasma corticosterone levels with two objectives: (i) to determine the effects of the exposure to the fearinducing paradigm during peripubertal period on females' corticosterone reactivity to an homotipic stressor, and in this way to validate the effectiveness of the paradigm involving an adaptation of the hypothalamic-pituitary-adrenal axis to the stressors, and (ii) to study whether corticosterone reactivity on p42 of the stressed females will be related to the effects of juvenile stress on adulthood. Behavioral observations of social interactions (resident-intruder test, cohabitation female-male and maternal aggression) were videotaped and the behavioral scoring was performed by an experimenter blind to treatment condition assisted by a computer program (The Observer 5.0.25, Noldus, 2003). Anxiety-like behavior displayed in the elevated plus maze (EPM; for details of the test see supplementary materials) and body weight (variables related to male aggression) were considered in order to distribute homogenously the males and females used as intruders (in the resident-intruder and maternal aggression tests) or as companions (for cohabitation female-male) between the two experimental groups.

\subsection{Peripubertal stress protocol}

We applied a peripubertal stress protocol (Cordero et al., 2012; Marquez et al., 2013) (Fig. 1A). Briefly, this protocol involves the exposure to two different fear-inducing stressors: i) the synthetic fox odor trimethylthiazoline (TMT) (Phero Tech Inc., Delta, BC, Canada), a synthetic version of a compound found in fox feces that evokes innate fear in rats; and (ii) exposure to an elevated platform (EP, $12 \mathrm{~cm} \times 12 \mathrm{~cm}$, elevated $95 \mathrm{~cm}$ from the ground) under direct bright light (470-500 lux). Each of these stressors lasted $25 \mathrm{~min}$. The stressors were applied in a semi-random order, and on an unpredictable schedule (a total of 7 days across p28-p42) during the light phase (see Fig. 1). Following each stress session, the animals were returned to their home-cages. During the first 15 min back in the home-cage, a transparent Plexiglas wall with holes was used to separate each of the animals in the cage. The control animals were handled on the days that their experimental counterparts were exposed to stress. Before starting the stress protocol, on day p28, all the animals from the stress group were
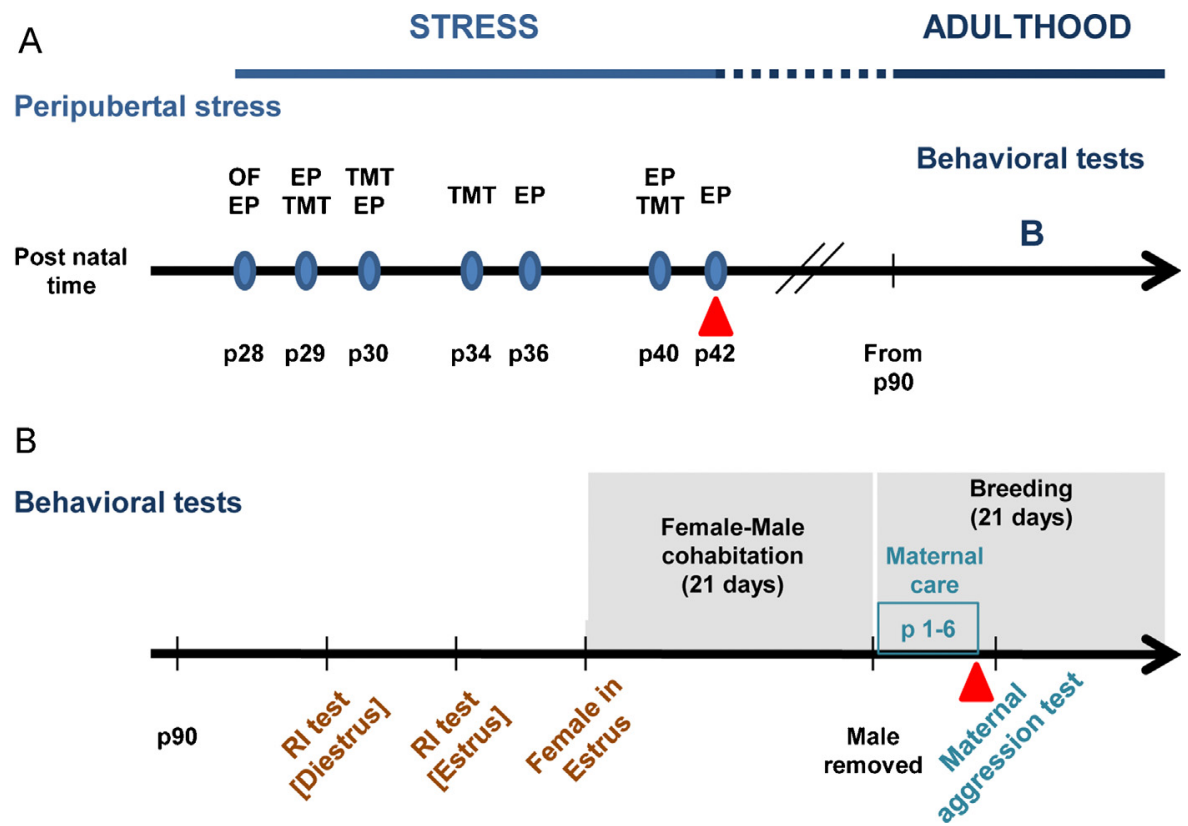

Figure 1 Fear-inducing experimental design $(\mathrm{A})$ and experimental procedures during adulthood (B), including the general experimental design and the sequence of behavioral tests performed on the females. OF, open field; EP, elevated platform; TMT, exposure to the predator odor trimethylthiazoline; $\Delta$ blood sampling; RI, resident-intruder test; $p$, postnatal day. 
exposed to a 5 min open field (rectangular arena; $50 \mathrm{~cm} \times 50 \mathrm{~cm})$.

\subsection{Resident-Intruder tests (RI)}

Female rats underwent the RI tests at the age of 3-4 months. The RI protocol was adapted from (Ho et al., 2001). The females were exposed to two RI tests (first RI during diestrus phase and second RI during estrus phase) separated for at least two complete estrus cycles, and intruders were used only one time. Briefly, thirty minutes before each RI test the other two cagemate females were removed from the resident's home-cage and only returned once the RI test had ended. In order to avoid behavioral interference between cagemates, only one female rat per cage was tested on one given day. RI tests were carried out during the beginning of the dark period (between $1930 \mathrm{~h}$ and $2130 \mathrm{~h}$ ). During the RI test, the resident control or peripubertal stressed female was exposed in its home-cage to a similar size, unfamiliar OVX female Wistar rat for $15 \mathrm{~min}$. The following parameters related to inter-female aggression were scored for residents: attack latency time, number of attacks, lateral threat, offensive upright and keep down. The percentage of time spent performing the latter four behavioral parameters were summarized as total aggressive behavior.

\subsection{Behavior during cohabitation with male partners}

To evaluate the effects of peripuberty stress exposure on females' aggressive behavior during cohabitation with a male partner, adult 3-months old naïve male Wistar rats were used as partners for the experimental subjects. Upon arrival, male rats were housed in groups of 3 per cage. Three weeks later, the males were caged for 21 days with a 3-4 month-old adult female in estrus (either control or peripubertal stressed, $N=12$ /gender/group). At the end of this period, the male was removed prior to female parturition.

During female-male cohabitation, the home-cage was changed 3 times (once per week) at approximately 1700 $1900 \mathrm{~h}$, and the animals were provided with a new cage with fresh sawdust. This arousing experience stimulates social interactions and for this reason, it was used as the starting time for behavioral observations (Cordero et al., 2012). The first time each female and male were put together in the home-cage, and the periods immediately following homecage changing on days 9 and 18, were video-recorded for $10 \mathrm{~min}$. The number of attacks and the time displaying defensive-submissive behavior (either freezing or being in a supine position under the other rat) were scored. Animals were not visibly wounded by these behaviors, except for superficial scars on a few females.

\subsection{Body weight gain during pregnancy and obstetric outcomes}

During the period of cohabitation with a male, female's body weight was monitored once per week (at the time the homecage was changed) and the percent of body weight gain during the total period of cohabitation was calculated. Subjects with late pregnancy (delivery of the pups $>7$ days after the end of the cohabitation period with the male) or not pregnant, were excluded from the analyses of body weight gain (late pregnancy: 1 control; No pregnancy, 1 stress; number of animals included in the final analyses, $N=11$ / group). For the following obstetric outcome analyses the exclusion criteria was no pregnancy ( 1 stress female; number of animals included in the final analyses: control, $N=12$; stress, $N=11$ ): cannibalism of pups and number of days elapsed between first encounter with the male and offspring delivery.

\subsection{Maternal aggression test}

The maternal aggression test was conducted on postpartum day 7 during the first two hours of the dark period. Dams and their litters were brought in their home-cage to a dimly lit and quiet behavioral observation room, and allowed to habituate during 15 min prior test. A smaller male intruder (175$190 \mathrm{~g}$ ) was then placed inside the cage opposite to the dam and litter, and the session was videotaped with a low light sensitivity video camera for 10 min session. A new inexperienced intruder male was used for each session. The sessions were closely observed for danger to the pups by either the male intruder or the dam, and if harm appeared imminent then the session was stopped and data from that session (with the exception of latency to attack) was not available for statistical analysis. Following testing, the male was removed from the cage, and the dam and pups were returned to the colony room. The following behaviors were scored for residents: percent time sniffing the opponent, attack latency time, number of attacks, lateral threat, offensive upright, and keep down. The percentage of time spent performing the latter four behavioral parameters were summarized as total aggressive behavior. Besides, percent time of maternal care during the test (dam is with the pups, while she licks pups, retrieves, or crouches over pups) was scored as well. Data from one control dam was not included in the analyses since the video file with the recorded behavior was corrupted and data was not available.

\subsection{Determination of estrous cycle phase}

The estrous cycle was monitored daily in all females from p90 until they were housed with a male. Female rats were lightly restrained manually and a swab wet with room temperature normal saline solution ( $\mathrm{NaCl} 0.9 \%$ ) was gently and shallowly inserted into the vagina and smeared on a new slide. The slide was immediately fixed with $1 \%$ toluidine blue solution and observed under the microscope $(10 \times$ and $40 \times$ objective lenses) to evaluate cells that characterize each stage of the estrous cycle (for details on the procedure, see Cordero et al., 2012).

\subsection{Plasma corticosterone, oxytocin and vasopressin assays}

To assess possible alterations of corticosterone reactivity on the last day of the stress protocol ( $p 42$ ), as a consequence of exposure to the fear inducing paradigm described above, blood samples were obtained from all stress females and a subset of control females $(N=12)$ only exposed to the EP on 
p42 and dismissed for the following experiment afterwards. The samples were collected at four time points [basal prior exposure to the elevated platform, immediately after 25-min of exposure to the EP ( 0 min), and 30- and 60-min after offset of the exposure to the EP]. One of the female rats submitted to the stress group fall from the EP and, consequently, samples from this rat were not collected. Furthermore, on postpartum day 7 th, during the first hour of the light cycle, blood samples were collected from the dams that were submitted to the maternal aggression test during the dark cycle on the same day, and corticosterone (CORT), oxytocin (OX) and vasopressin (AVP) plasma levels were assessed. All the blood samples were collected by tail-nick that allows taking blood samples at different time points from the same animal in order to study hormonal dynamics. Tail-nick procedure consisted of gently wrapping the animals with a cloth, making a 2-mm incision at the end of one of the tail arteries and then massaging the tail while collecting, within 2-min, $300 \mu \mathrm{l}$ of blood into ice-cold heparin capillary tubes (Microvett cb300, Sarsted, Switzerland). When several samples were collected on the same day (i.e., on p42), the second and following samples were obtained by gently removing the scab from the first incision. The tubes were centrifuged at $4{ }^{\circ} \mathrm{C}$ (4500 rpm $\left.\times 20 \mathrm{~min}\right)$, and plasma aliquots for corticosterone assays were stored at $-20{ }^{\circ} \mathrm{C}$ until the assay was performed. In plasma aliquots for oxytocin and vasopressin assays, aprotinin (a protease inhibitor; Sigma, 0.039 trypsin inhibitor units in $10 \mu \mathrm{l}$ per sample) was added to the aliquots and stored at $-20{ }^{\circ} \mathrm{C}$. Plasma corticosterone (Correlate-EIA ${ }^{\mathrm{TM}}$ Corticosterone), OX (Correlate-EIA ${ }^{\mathrm{TM}}$ Oxytocin) and AVP (Correlate-EIA ${ }^{T M} \mathrm{arg}^{8}$-Vasopressin) levels were measured using commercial enzymatic immunoassay kits (Assay Designs Inc., USA) according to the supplier's recommendations.

\subsection{Statistics}

The SPSS 17.0 (SPSS, Chicago, IL) statistical package was used for the statistical analyses. The normality and homogeneity of variance of the data were tested, and the adjusted statistics were used as required. Parametric statistics: mean comparisons were carried out with either oneway and Student's $t$-tests (group: control vs. stress) or factorial ANOVAs (with group as between subject variable). If Levene's test for equality of variances was significant, the statistics for equal variances not assumed is reported with the altered degrees of freedom. Area under the curve (AUC) values of plasma corticosterone was calculated by the trapezoid rule (area under the polygon formed by the connecting time values between times of sampling). Variables related to study whether corticosterone reactivity on p42 of the stressed females will be related to the effects of juvenile stress on adulthood were analyzed by factor analysis, using a principal components solution (PCA) with a varimax orthogonal rotation of the factor matrix. The acceptance criteria for emerging components was conservative; following a PCA, only factors with eigenvalues larger than 1.0 were accepted; accordingly, factors with high-loading scores $(>0.60)$ were considered as relevant contributing factors. Correlational analyses between hormonal levels and behavioral measures were conducted by Pearson's test with normally distributed data, or Spearman correlation with not normally distributed data. Paired samples analyses between siblings were performed with Wilcoxon. Frequency distributions and percentages by strata $(<30 \mathrm{~s},>30 \mathrm{~s})$ were calculated for the parameter of latency to attack during the maternal aggression test and litter size, and differences between groups were analyzed by generating Kaplan-Meier curves and survival analysis. $p$ value $<0.05$ was accepted as significant.

\section{Results}

\subsection{Corticosterone reactivity to the elevated platform on day $\mathrm{p} 42$}

Samples were taken on p42 before and immediately, 30 and $60 \mathrm{~min}$ after a 25-min exposure to the elevated platform (EP). Compared to control females (that were exposed to this stressor for the first time), stress females had reduced corticosterone area under the curve (Fig. S1, Table S1; $Z=-2.71, p<0.007)$ and significantly lower corticosterone levels at 30 -min after offset of the EP exposure $(Z=-3.32$, $p<0.001)$. No significant differences were found between the groups in basal corticosterone levels. These results resemble the data we obtained from males exposed to the same stress procedure during the peripubertal period (Marquez et al., 2013), suggesting that males and females have a similar adaptation in the glucocorticoid response to repeated stress exposure during the peripubertal period.

\subsection{Aggressive behavior against female intruders at adulthood}

Stress females were more aggressive than control females when confronted to OVX female intruders in both estrus cycles (Fig. 2A and Table S2): diestrus (D: total frequency of attacks, $t=-3.23, \mathrm{df}=12.5, p<0.01$; and total frequency offensive behavior, $t=-3.01, \mathrm{df}=13.9, p<0.01$ ) and estrus (E: SQRT of total frequency of attacks, $t=-2.2$, $\mathrm{df}=22, p<0.04$; and SQRT of total frequency offensive behavior, $t=-2.63$, df $=22, p<0.02$ ). No significant differences were found between the groups in the latency of the first attack in any of the estrus cycles (Table S2: D: SQRT latency attack, $t=0.45$, $\mathrm{df}=22$, n.s.; $\mathrm{E}: \mathrm{Z}=-1.11$, n.s.). Exposure to stress during puberty did not affect the time sniffing/exploring the intruder, in neither of the RI tests (D: control $=19.83 \pm 2.4 ;$ stress $=18.95 \pm 2.4 ; t=0.26, \mathrm{df}=22$, n.s.; $\quad$ E: $\quad$ control $=12.26 \pm 1.57 ; \quad$ stress $=11.14 \pm 1.21$; $t=0.53, \mathrm{df}=22$, n.s. $)$.

\subsection{Female-male cohabitation}

On the first day of cohabitation with an unfamiliar male partner, stress females displayed higher frequency of aggressive behavior against the male partner than control females did (Fig. 2B, Table S3, $t=-2.34$, df $=14.53, p<0.03$ ). Similar to the results obtained in the RI tests, no significant differences were found between control and stressed females in the latency to attack (control $=415.21 \pm 101.57$, 101.57, stress $=278.27 \pm 92.08: Z=-1.07$, n.s. ), or percent time sniffing/exploring the male rat (control $=7.88 \pm 0.93$, stress $=6.82 \pm 1.46, \quad Z=-1.33$, n.s.). Furthermore, the increase of aggression of the stress females against the male 
A

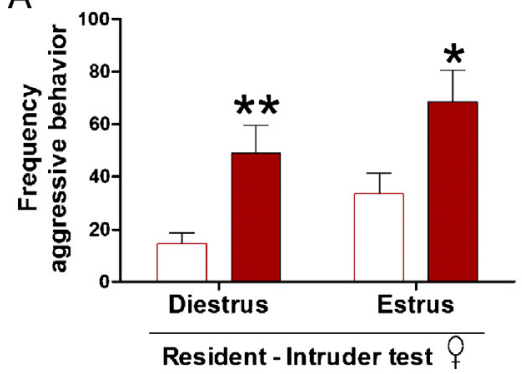

B

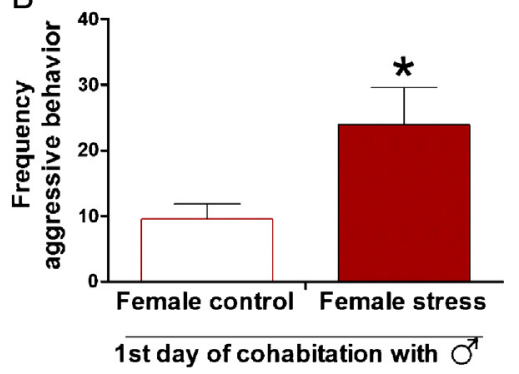

Figure 2 Effects of peripubertal stress on non-maternal related aggression tests. Peripubertally stressed females displayed higher frequency of aggressive behavior during RI tests in both estrus phases (A), and against a male partner as evaluated during the first 10min of the cohabitation (B). The results are the mean + SEM. ${ }^{*} p<0.05$; ${ }^{* *} p<0.01$ vs. control group.

partners was restricted to the first day of cohabitation (Table S3).

On the other hand, male partners of stress females spent a higher amount of time displaying aggressive behaviors than control males across the cohabitation period (Fig. 3A, Table S3: males' total percent time of aggressive behavior, $Z=-2.19, \quad p<0.03 ; \quad D 1, \quad Z=-2.32, \quad p<0.02 ; \quad D 9$, $Z=-0.03$, n.s.; D18, $Z=-2.32, p<0.02)$. Stress females spent significantly higher percent of time in submissivedefensive behavior than control females (Fig. 3B, Table S4: $Z=-2.25, p<0.03)$. Furthermore, the average of latency to display submissive behavior during the scoring days was shorter for stress females than control females (control $=292.75 \pm 44.7 ; \quad$ stress $=175.26 \pm 39.25 ; \quad Z=-2.14$, $p<0.033$ ), even though no significant differences were found between the groups in the latency to display aggressive behavior by the males against their partners (control $=349.68 \pm 64.9 ; \quad$ stress $=256.1 \pm 45.1 ; \quad t=1.17$, $\mathrm{df}=22$, n.s. $)$.

No significant correlations were found between the percent time of females' aggressive behavior on the first day of cohabitation and the percent time of males' aggressive behavior during (i) the first day of cohabitation (all, $r=0.26$, n.s.; control, $r=0.16$, n.s.; stress, $r=0.1$, n.s.), or (ii) the average of aggressive behavior of the male during the cohabitation period (all, $r=0.27$, n.s.; control, $r=0.18$, n.s.; stress, $r=0.01$, n.s.). However, the percent time of females' defensive behavior during the first day was significantly correlated with the percent time of males' aggressive behavior during (i) the first day of cohabitation (all, $r=0.68, p<0.001$; control, $r=0.94, p<0.001$; stress, $r=0.6, p<0.05$ ), and (ii) the average of percent time of males' aggressive behavior during the cohabitation period for all the animals, and control group but not for the stress group (all, $r=0.5, p<0.02$; control, $r=0.68, p<0.02$; stress, $r=0.3$, n.s. ). A plausible explanation for the lack of correlation between the offensive behavior of males and females during the first encounter could be due to the fact that all the females were in estrus during the first encounter, and therefore, the offensive behavior displayed by males and females during the first encounter contains possibly a mixture of sexual and aggressive behaviors.

\subsection{Sibling analyses of the effects of stress during puberty on non-maternal related aggressive behavior}

Since in our experimental design, 11 pairs of sisters were distributed between the two groups we compared the nonmaternal related aggressive behaviors displayed in the different behavioral tests between sisters submitted to either the control or the peripuberty stress conditions. We found that stress females inflicted a higher number of attacks (nonparametric related samples: Wilcoxon signed ranks test, $D$ : $\mathrm{Z}=-2.13, p<0.033 ; \mathrm{E}: \mathrm{Z}=-2.22, p<0.026)$ and higher total frequency of aggressive behavior (Fig. S2, for both, D
A

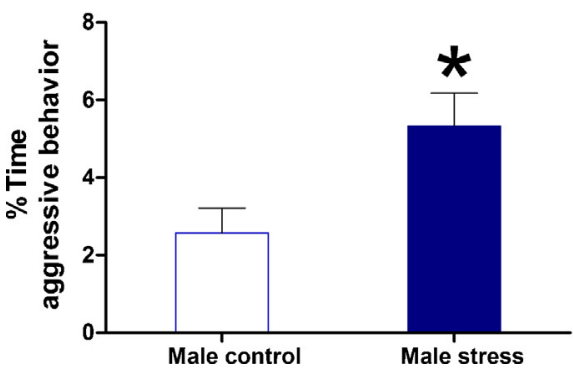

B

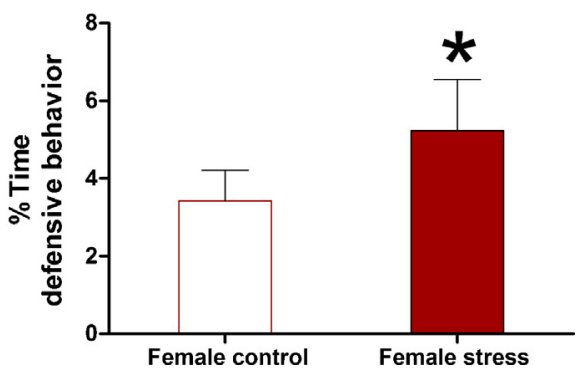

Figure 3 Aggressive and defensive behaviors during female-male cohabitation. During the 21 days of female-male cohabitation between pairs of rats involving either control or peripubertally stressed females ( $N=12$ /group), interactions were recorded once per week (on the 1st, 9th and 18th cohabitation days) for $10 \mathrm{~min}$. (A) Males cohabitating with peripubertally stressed females show higher aggression levels against their partners than males cohabitating with female control rats. (B) During cohabitation with a male, stressed females displayed a higher defensive behavior than control females. The results are the mean + SEM. ${ }^{*} p<0.05$ vs. control group. 
and $\mathrm{E}, \mathrm{Z}=-2.13, p<0.033$ ) to the OVX intruders during the Resident-Intruder tests than did their sisters in the control group. Furthermore, stress females showed a higher frequency of aggressive behaviors toward the male partner during the first day of cohabitation than their sisters in the control group did (Fig. S2, $Z=-1.96, p<0.05$ ). Thus, in every test, stress females were more aggressive than their respective sisters in the control group (Chi-square analyses of the number of females of one group that were more aggressive than their respective sister on the other group: D_RI and D1 cohabitation: of 11 pairs of siblings, 2 females in the control and 9 females in the stress group were more aggressive than their siblings in the opposite group, $\chi^{2}(1$, $N=11)=4.45, p<0.035$; E_RI: 1 female in the control and 10 females in the stress group were more aggressive than their siblings in the opposite group, $\chi^{2}(1, N=11)=7.36$, $p<0.007)$.

\subsection{Maternal aggression}

On postpartum day 7th, all the dams with litter (except one control dam that had a late delivery and was not included in this part of the study) were submitted to the maternal aggression test. Note that this test requires that the female has offspring alive at the time of the test, therefore females that (i) cannibalized their pups (1 dam from the control group and 2 dams from the stress group), (ii) were not pregnant ( 1 female from the stress group) or (iii) had a late pregnancy ( 1 female from the control group) were not submitted to the maternal aggression test. The final number of animals included in the analyses was 9 per group. Given that the remaining females included in the analyses were not matched siblings, siblings' analyses were not performed on this test. Due to the violence of the attacks that imperiled the safety of the offspring, the test was stopped in a few cases ( 2 control and 3 stressed dams) in which the only data collected was the latency to attack the intruder, as this parameter allowed us to include data from all animals submitted to the test (for details and analyses on other variables see supplementary material Fig. S3). Compared to control dams, stressed dams showed shorter latencies to attack the intruder during the maternal aggression test (Fig. 4A, Kaplan-Meier survival analysis, Log Rank [Mantel-Cox: $\chi^{2}=9.58, p<0.002$; Breslow (generalized Wilcoxon): $\chi^{2}=8.98, p<0.003$; Tarone-Ware: $\chi^{2}=9.41$, $p<0.002$ ]. No significant differences were found between the groups on the time the dams spent with the pups during the test (Fig. S3B-D).

\subsection{Body weight gain during pregnancy, obstetric outcomes and postpartum hormones}

\subsubsection{Body weight gain during pregnancy and obstetric outcomes}

Kaplan-Meier survival analysis showed that compared to control dams, stressed dams had a lower number of pups [Fig. S4, Kaplan-Meier survival analysis, Breslow (generalized Wilcoxon): $\chi^{2}=4.13, \quad p<0.05$; Tarone-Ware: $\chi^{2}=4.19, \quad p<0.05 ;$ Log Rank (Mantel-Cox: $\chi^{2}=4.1$, $p<0.05)$ ]. Furthermore, considering the number of pups per litter, there were more stress dams with less than 9 pups per litter (average number of pups of the whole population) than control (Fig. S4A, control, $N=1 / 12$; stress, $N=6 / 12 ; \chi^{2}$ $(1, N=24)=5.04, p<0.025$, Fisher's exact test $p<0.034)$.

There were no significant differences between the groups on (i) body weight gain during pregnancy (control females $=125.84 \pm 2.1, \quad$ stress $\quad$ females $=126.77 \pm 1.8$; $t=-0.34$, df $=20$, n.s.), (ii) number of dams that cannibalized their litter $\left(\right.$ control $=1 / 12$; stress $=2 / 11 ; \chi^{2} \quad(1$, $N=23$ ) $=0.49$, n.s.), (iii) number of days elapsed between first encounter with the male partner and offspring delivery (control $=23 \pm 0.8$; stress $=22 \pm 0.2 ; Z=-0.5$, n.s.); and (iv) weight of the male and female offspring at weaning (male offspring: control $=53.97 \pm 2.04$; stress $=57.32 \pm 3.35 ; t=-0.89, \mathrm{df}=18$, n.s.; female offspring: control $=51.66 \pm 2.1, \quad$ stress $=54.2 \pm 2.9 ; \quad t=-0.73$, $\mathrm{df}=18$, n.s. $)$.

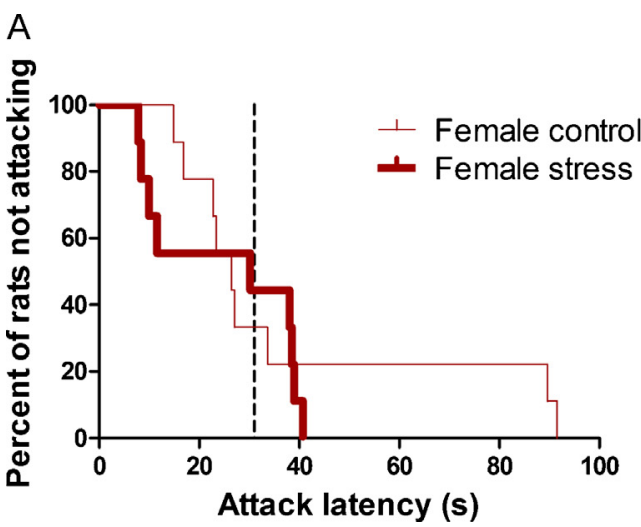

B

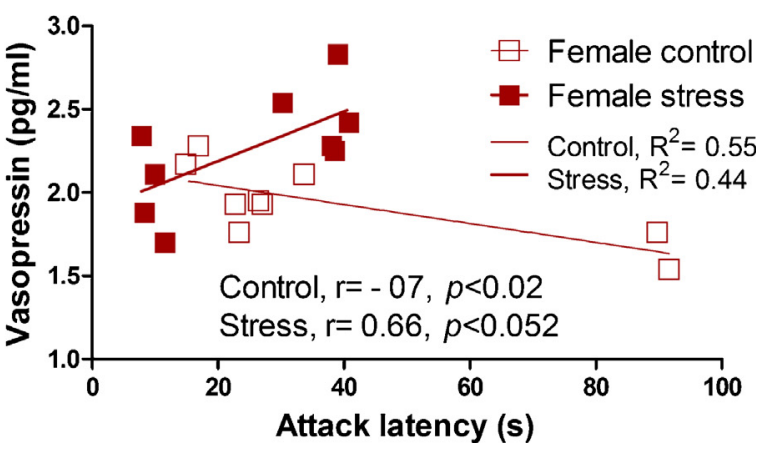

Figure 4 Effects of peripubertal stress on maternal aggression against a male intruder. (A) Kaplan-Meier survival plot of latency to attack the intruder, and (B) correlation between plasma vasopressin at post-partum day 7 and latency to attack the intruder during the maternal aggression test. Compared to control dams, stressed dams showed shorter attack latencies during the maternal aggression test [Kaplan-Meier survival analysis, Log Rank (Mantel-Cox: $\chi^{2}=9.58, p<0.002$; Breslow (generalized Wilcoxon): $\chi^{2}=8.98$, $p<0.003$; Tarone-Ware: $\chi^{2}=9.41, p<0.002$ ]. Latency to attack the intruder during the maternal aggression test correlated with vasopressin levels at post-partum day 7; the correlations were of opposite direction for control $(r=-0.7, p<0.02)$ and stress $(r=0.66$, $p<0.052)$ groups $(N=9$ /group $)$. 

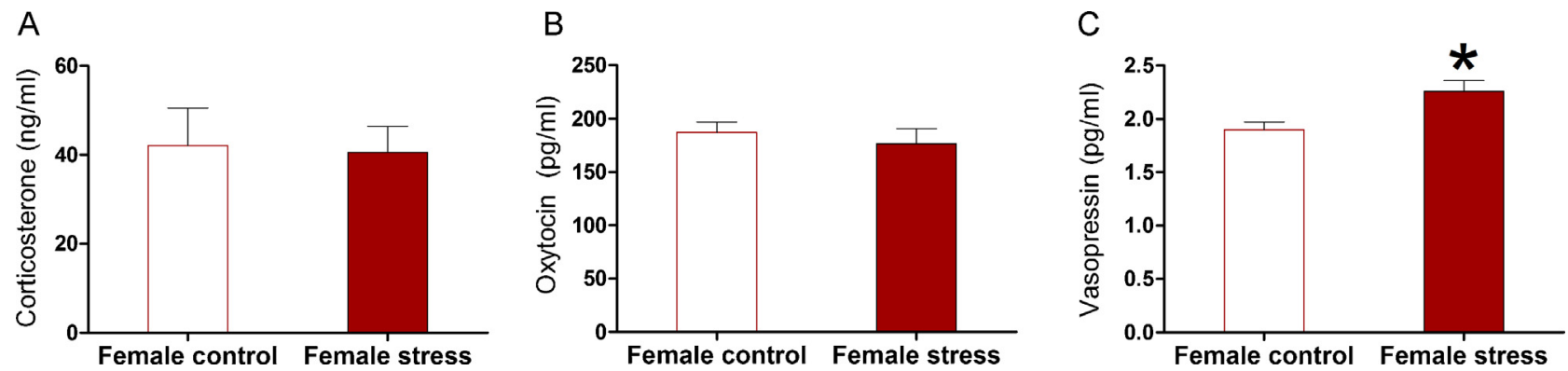

Figure 5 Effects of peripubertal stress on circulating hormones. Levels of plasma corticosterone (A), oxytocin (B) and vasopressin (C) on post-partum day 7 were compared between control and stress females. Stress dams had higher levels of plasma vasopressin than controls (C). $N=10-9 /$ group. The results are the mean + SEM. ${ }^{*} p<0.05$ vs. control group.

\subsection{Postpartum hormonal levels}

Blood samples were drawn from dams on postpartum day 7th, and the plasma levels of corticosterone, oxytocin and vasopressin were measured (Fig. $5 \mathrm{~A}-\mathrm{C}$ ). Only dams that were subjected to the maternal aggression test were included in the analyses. Stress females had higher vasopressin levels than control females $(t=-2.7, \mathrm{df}=17, p<0.015)$. No significant differences were found between the groups in the levels of corticosterone $(t=0.158, \mathrm{df}=17, \mathrm{n} . \mathrm{s}$. $)$ or oxytocin $(Z=-0.98$, n.s. $)$.

\subsection{Factor analysis of hormonal and behavioral post-stress outcomes}

In order to explore whether corticosterone reactivity at p42 (last day of the peripuberty stress protocol) would be related to the impact of stress during puberty on aggressive behavior [both, average of non-maternal related aggression (see Fig. S5 for details), and maternal aggression] and endocrine outcomes in the adulthood, a factorial analysis including these variables was conducted. Note that these analyses can only be carried out in animals submitted to the peripuberty stress protocol, as they were the only ones submitted to the elevated platform at $\mathrm{p} 42$.

Seven factors emerged in the principal component analysis, the first two, accounting for $72 \%$ of the variance, are shown in Table 1 for further consideration. Factor 1 (eigenvalue $=2.9$ ) accounted for $41 \%$ of the variance and consisted of the following high-loading variables: (i) increased corticosterone levels 30 min after exposure to stressor (p42), (ii) decreased aggressive behavior (both, non-maternal and maternal related aggressive behavior), and (iii) increased postpartum AVP levels. Interestingly, adaptation of corticosterone reactivity to repeated stress during puberty loaded in the same factor as the main effects of peripuberty stress on aggressive behavior and postpartum hormonal levels.

Factor 2 (eigenvalue $=2.2$ ) accounted for $31 \%$ of the variance and consisted of the following high-loading variables: (i) increased total corticosterone reactivity (area under the curve) to stressor exposure (p42); (ii) increased non-maternal aggressive behavior, (iii) increased corticosterone and decreased oxytocin post-partum levels.

\subsection{Relationship between aggressive behavior and postpartum hormonal levels}

Given the relationships between aggressive behavior and postpartum hormonal levels revealed by the factorial analyses done for the stress group, we performed correlational analyses for control and stress groups independently to further explore whether the relationship between aggressive behavior and postpartum hormonal levels was affected by the exposure to stress during puberty. Interestingly, significant (2-tail) correlations were found for control and stress group but in the opposite direction between (i) AVP and latency to attack the intruder during the maternal aggression test (Fig. 4B: control, $r=-0.7, p<0.02$; stress, $r=0.66$,

Table 1 Principal component analyses: stress female group.

\begin{tabular}{llrr}
\hline Variables & & Factor 1 (41\%) & Factor 2 (31\%) \\
\hline CORT reactivity p42 & AUC total & 0.64 \\
& Corticosterone 30 min & 0.85 & 0.66 \\
Aggressive behavior & Av. Freq. Attacks RI-D, RI-E, COH-D1 & -0.61 & 0.89 \\
& Latency to attack maternal aggression test & 0.92 & 0.88 \\
Postpartum hormones & AVP & & -0.62 \\
& CORT & & \\
& OXT & & 0.62 \\
\hline
\end{tabular}

Emergent components in principal component analysis and the accompanying high-loading factors of hormonal and behavioral post-stress outcomes. Factor loadings higher than 0.6 produced by a varimax rotation are shown for each factor. The percentage of the total variance accounted for by each factor is given in parentheses. 
$p<0.052$ ) and (ii) CORT and non-maternal aggressive behavior (Fig. S6: control, $r_{\mathrm{s}}=-0.85, p<0.01$; stress, $r_{\mathrm{s}}=0.8$, $p<0.02)$.

\section{Discussion}

In this study, we present clear evidence that exposure to stressful experiences during puberty increased female rats' aggressive behaviors at adulthood both against females and males. Increased frequency of aggression toward females was observed, both during diestrus and estrus. Increased frequency of aggression toward the male partner was specifically observed during their first encounter (females in estrus). In fact, when female-male interactions were assessed throughout the cohabitation period, male partners of stress females were more aggressive (percent time) against their partners than male partners of control females were, and stress females displayed higher defensive/submissive behavior (percent time) than female controls did. Furthermore, exposure to puberty stress increased females' maternal aggression against a male intruder (lower latency to attack the intruder) and postpartum plasma levels of AVP.

Several rodent models have been proposed to study the development of human aggressive behavior, but few of them have included non-lactating females, despite the fact that both similarities and differences have been reported in the way life experiences influence the development of aggressive behavior on males and females. For instance, in mice increased aggressive behavior was found in both males and females, after isolation (Malick, 1979; More, 2008); while effects of prenatal stress on aggressive behavior in the offspring have been found to be sex-dependent, increased in females (Vom Saal et al., 1991), and reduced in males (Kinsley and Svare, 1986). In rats, sex-dependent effects of stress on aggressive behavior have been reported as well, for example after immobilization stress females increase aggressive behavior (Albonetti and Farabollini, 1993b) while males become less aggressive (Albonetti and Farabollini, 1993a). These findings caution against the generalization of the results between genders and emphasize the need to evaluate animal models to study the development of aggressive behaviors in females as well as in males.

We have previously reported that males submitted to fearinducing experiences around the time of puberty become more aggressive against other conspecifics males and females (Cordero et al., 2012; Marquez et al., 2013). In the current study, we have found that exposing female rats to the same fear-inducing protocol during puberty increases their aggressive behavior in adulthood, independent of the estrus cycle phase. Not only were the stress females more aggressive than controls against OVX females. When confronted for the first time with an unfamiliar male (40-50\% larger than themselves) during the estrus (receptive) phase, stress females were also more aggressive against the male than control females. We have previously reported a similar finding for males submitted to the same peripuberty stress protocol: compared to control males, stress males were more aggressive not only against different types of male intruders including some 25\% larger than themselves (Marquez et al., 2013) - but also against females (Cordero et al., 2012). Our results are in contrast to observations in hamsters where juvenile stress enhances offensive aggression toward smaller intruders and decreases aggression toward intruders of equal size (Delville et al., 1998); the effects of stress during the peripubertal period on aggressive behavior in rats do not depend on the sex or difference in size between the opponents. However, a difference between females and males submitted to peripubertal stress was that the stress males were more aggressive than control males against their female companions for the total duration of the cohabitation (Cordero et al., 2012); yet, in the current study, stress females only displayed an increase of aggression against the male companion during the first day of cohabitation. The difference in size between female-male dyads clearly favored the male to defeat and submit the female companion. Interestingly, even though no differences in aggressive behavior were found between control and stress females after the first day of cohabitation, male partners of stress females displayed higher aggression against their partners than male partners of control females. Since males were balanced across groups regarding their body weight and anxiety-like behavior (variables related to male aggression), differences in the aggressive behavior of the males against the female partners suggest deficits in the nature of the social interactions of stress females with their male partners. As a consequence of the higher aggression received, stress females displayed higher percent time showing submissive/defensive behavior than control females did.

It is noteworthy that antisocial and conduct disorders in women have been associated with a high risk of having been involved in mutually violent relationships (Bardone et al., 1996; Lewis et al., 1991). Interestingly, in a study with a sample of men in treatment for intimate partner violence it was reported that $40 \%$ had partners who initiated the violence (Gondolf, 1996). In humans, perpetration of intimate partner violence has been related to personality disorders in men (Edwards et al., 2003), and more recently in women (Walsh et al., 2010). Furthermore, exposure to childhood trauma increases the risk to develop both, personality disorders and intimate partner violence (Beauchaine et al., 2009).

We further found that peripuberty stressed females showed enhanced maternal aggression against a male intruder introduced in their homecage on postnatal day 7. Lactating females are known to be more aggressive than virgin females against a male intruder, and the increase of aggressive behavior during the lactating period has been related to changes in the OXT and AVP systems that begins during pregnancy and reaches the peak following maternal experience (Caughey et al., 2011). Importantly, stress dams had higher AVP - but not OXT - plasma levels than controls, as evaluated in postpartum day 7. Besides the effects on maternal aggression, AVP has been associated with aggressive behavior in several studies. For example, a positive correlation between aggressive behavior and vasopressin levels in the cerebrospinal fluid have been reported for rats (Haller et al., 1996) and violent humans (men and premenopausal women) with personality disorders (Coccaro et al., 1998). In male rats, distinct correlations were found between vasopressin release in the lateral septum and bed nucleus of the stria terminalis and aggressive behavior displays (Veenema et al., 2010). Furthermore, AVP treatment increases brain activity in aggression-related areas (Ferris, 2008), and conversely, antagonists of AVP receptors $1 \mathrm{~A}$ were found to 
reduce aggression while simultaneously suppressing the pattern of brain activation caused by an intruder (Ferris, 2008).

Given the above mentioned literature and our own findings showing an increase of AVP and aggressive behavior in stressed females, our results showing opposite intragroup correlations between AVP and maternal aggression (positive in control and negative in stress group) suggests a different role of AVP on maternal aggression depending on life experiences. Interestingly, the factorial analyses performed in the stress group showed that postpartum AVP levels loaded in the same factor as maternal and non-maternal related aggression but inversely (i.e., the lower AVP, the higher the aggression). The only study to our knowledge (Gutzler et al., 2010) that investigated the role of AVP in non-maternal related aggression in female rodents found that injection of AVP, and the $\mathrm{V} 1 \mathrm{a}$ antagonist, into the anterior hypothalamus reduces and increases, respectively, offensive aggression in female hamsters; in contrast to the opposite effects that the same treatments induce in males. A similar AVP sex-dependent effect has been reported in humans (Thompson et al., 2006), in men intranasal administration of AVP decreases perception of friendliness in the faces of unfamiliar men, while the same treatment in women induces the opposite effect. These data suggest a complex gender-dependent interaction between AVP levels and aggression.

The validity of peripheral measurement of OXT and AVP to reflect central processes related to behavior is still under question; arguments against arise from (i) their specific function in behavior which seems to be dependent on different patterns of neuropeptides synthesis, release and receptor distribution (Bosch and Neumann, 2012); and (ii) even though their major source is the brain, they are synthesized peripherally as well (uterus, gonads, heart and thymus). Accordingly some studies have shown differential central and peripheral release of the peptides under certain conditions, in humans (Kagerbauer et al., 2013) and in rodents (Wotjak et al., 1998). On the other hand, several studies have shown a relation between peripheral concentration levels and behaviors controlled centrally (Fries et al., 2005; Strathearn et al., 2012).

Taking into account that human studies have often found a high prevalence of psychopathology among first-degree relatives, and impulsive aggression is highly heritable (Seroczynski et al., 1999) and that boys and girls often shared with siblings traits known to convey potential risk factors for youth violence, i.e., hyperactivity, impulsivity and sensation seeking (Laporte et al., 2011; Zanarini et al., 2004), at weaning during the formation of the groups, we submitted sisters from different litters to a different experimental condition, control or stress, in order to study the potential role of the stress in exacerbating pre-existent high aggressive behavior. We observed that aggressive behavior was increased in siblings submitted to the stress condition, and that in most of the cases females with the higher scores in aggressive behavior in the control group were the siblings of females that presented the higher scores in the stress group. Human studies have shown the relevance of genetic and environmental interaction for the development of violent behavior, i.e., it has been reported a high concentration of criminality within families (for review, Farrington et al., 2001), and an increased susceptibility of developing violent and antisocial behavior in the adulthood of children of violent parents growing up in stressful environments (for review, Koenen, 2005). Similar gene $x$ environment interaction was shown in female subjects (Ducci et al., 2008).

The developing adolescent brain is considered highly vulnerable to experiential input (Paus et al., 2008). Pubertal hormones, particularly, glucocorticoid levels have organizational effects on the adolescent brain and influence the maturation of adult social behaviors, such as programing the adult expression of agonistic behaviors. We show here that exposure to stressful situations during the peripuberty period in female rats leads to enhanced aggressive behaviors at adulthood under a variety of circumstances. Our findings are in agreement with an increasing number of studies in humans showing that the probability to suffer personality disorders accompanied by aggression is increased by exposure to adverse experiences during childhood for both, boys (Johnson et al., 1999) and girls (Foy et al., 2012; Johnson et al., 1999). We provide here an animal model, devoid of cultural influences and where aggressive behavior could not be accounted for by social learning, strongly supporting a role for biological factors in the development of aggressive behaviors following exposure to stressful events at puberty in females and allowing the possibility of tackling the underling neurobiological mechanisms and developing new treatments.

\section{Role of the funding sources}

The funding sources have contributed to the development of the research but do not have any interest in the results. They have not participated in the specific design of experiments and are not involved in the exploitation of the obtained data.

\section{Conflict of interest}

The authors declare not to have any conflict of interest.

\section{Acknowledgments}

We thank Coralie Siegmund, Olivia Zanoletti and Nicolas Perret for their excellent technical assistance. This work was supported by grants from the Swiss National Science Foundation (310000-120791 and 31003AB-135710; Sinergia CRSIK3122691; and the NCCR 'The synaptic basis of mental diseases'), Oak Foundation, and intramural funding from the EPFL.

\section{Appendix A. Supplementary data}

Supplementary material related to this article can be found, in the online version, at doi:10.1016/j.psyneuen.2013.07.005.

\section{References}

Albert, D.J., Walsh, M.L., 1984. Neural systems and the inhibitory modulation of agonistic behavior: a comparison of mammalian species. Neurosci. Biobehav. Rev. 8, 5-24.

Albonetti, M.E., Farabollini, F., 1993a. Restraint increases both aggression and defence in female rats tested against same-sex unfamiliar conspecifics. Aggress. Behav. 19, 369-376. 
Albonetti, M.E., Farabollini, F., 1993b. Effects of single and repeated restraint on the social behavior of male rats. Physiol. Behav. 53, 937-942.

Bardone, A.M., Moffitt, T.E., Caspi, A., Dickson, N., Silva, P.A., 1996. Adult mental health and social outcomes of adolescent girls with depression and conduct disorder. Dev. Psychopathol. 8, 811-829.

Beauchaine, T.P., Klein, D.N., Crowell, S.E., Derbidge, C., GatzkeKopp, L., 2009. Multifinality in the development of personality disorders: a Biology $\times$ Sex $\times$ Environment interaction model of antisocial and borderline traits. Dev. Psychopathol. 21, 735-770.

Blakemore, S.J., 2012. Development of the social brain in adolescence. J. R. Soc. Med. 105, 111-116.

Blanchard, R., Kleinschmidt, C., Fukunaga-Stinson, C., Blanchard, D.C., 1980. Defensive attack behavior in male and female rats. Anim. Learn. Behav. 8, 177-183.

Blanchard, R.J., Hori, K., Flannelly, K., Blanchard, D.C., 1987. The effects of ethanol on the offense and defensive behaviors of male and female rats during group formation. Pharmacol. Biochem. Behav. 26, 61-64.

Boccia, M.L., Pedersen, C.A., 2001. Brief vs. long maternal separations in infancy: contrasting relationships with adult maternal behavior and lactation levels of aggression and anxiety. Psychoneuroendocrinology 26, 657-672.

Bosch, O.J., Neumann, I.D., 2012. Both oxytocin and vasopressin are mediators of maternal care and aggression in rodents: from central release to sites of action. Horm. Behav. 61, 293-303.

Bosch, O.J., Pfortsch, J., Beiderbeck, D.I., Landgraf, R., Neumann, I.D., 2010. Maternal behaviour is associated with vasopressin release in the medial preoptic area and bed nucleus of the stria terminalis in the rat. J. Neuroendocrinol. 22, 420-429.

Caughey, S.D., Klampfl, S.M., Bishop, V.R., Pfoertsch, J., Neumann, I.D., Bosch, O.J., Meddle, S.L., 2011. Changes in the intensity of maternal aggression and central oxytocin and vasopressin $\mathrm{V} 1 \mathrm{a}$ receptors across the peripartum period in the rat. J. Neuroendocrinol. 23, 1113-1124.

Coccaro, E.F., Kavoussi, R.J., Hauger, R.L., Cooper, T.B., Ferris, C.F., 1998. Cerebrospinal fluid vasopressin levels: correlates with aggression and serotonin function in personality-disordered subjects. Arch. Gen. Psychiatr. 55, 708-714.

Cordero, M.I., Poirier, G.L., Marquez, C., Veenit, V., Fontana, X., Salehi, B., Ansermet, F., Sandi, C., 2012. Evidence for biological roots in the transgenerational transmission of intimate partner violence. Transl. Psychiatr. 2, e106.

de Kloet, E.R., 2010. From vasotocin to stress and cognition. Eur. J. Pharmacol. 626, 18-26.

DeBold, J.F., Miczek, K.A., 1984. Aggression persists after ovariectomy in female rats. Horm. Behav. 18, 177-190.

Delville, Y., David, J.T., Taravosh-Lahn, K., Wommack, J.C., 2003. Stress and the development of agonistic behavior in golden hamsters. Horm. Behav. 44, 263-270.

Delville, Y., Melloni Jr., R.H., Ferris, C.F., 1998. Behavioral and neurobiological consequences of social subjugation during puberty in golden hamsters. J. Neurosci. 18, 2667-2672.

Ducci, F., Enoch, M.A. , Hodgkinson, C., Xu, K., Catena, M., Robin, R.W., Goldman, D., 2008. Interaction between a functional MAOA locus and childhood sexual abuse predicts alcoholism and antisocial personality disorder in adult women. Mol. Psychiatr. 13, 334-347.

Edwards, D.W., Scott, C.L., Yarvis, R.M., Paizis, C.L., Panizzon, M.S., 2003. Impulsiveness, impulsive aggression, personality disorder, and spousal violence. Violence Vict. 18, 3-14.

Farrington, D.P., Jolliffe, D., Loeber, R., Stouthamer-Loeber, M., Kalb, L.M., 2001. The concentration of offenders in families, and family criminality in the prediction of boys' delinquency. J. Adolesc. 24, 579-596.

Ferris, C.F., 2008. Functional magnetic resonance imaging and the neurobiology of vasopressin and oxytocin. In: Neumann, I.D., Landgraf, R. (Eds.), Progress in Brain Research. Elsevier, Oxford, pp. 305-320.
Foy, D.W., Ritchie, I.K., Conway, A.H., 2012. Trauma exposure, posttraumatic stress, and comorbidities in female adolescent offenders: findings and implications from recent studies. Eur. J. Psychotraumatol. 3, 31.

Fries, A.B., Ziegler, T.E., Kurian, J.R., Jacoris, S., Pollak, S.D., 2005. Early experience in humans is associated with changes in neuropeptides critical for regulating social behavior. Proc. Natl. Acad. Sci. U. S. A. 102, 17237-17240.

Gondolf, E.W., 1996. Characteristics of Batterers in a Multi-site Evaluation of Batterer Intervention Systems. , http://www.mincava.umn.edu/documents/gondolf/batchar.html\#idp37612192.

Gutzler, S.J., Karom, M., Erwin, W.D., Albers, H.E., 2010. Argininevasopressin and the regulation of aggression in female Syrian hamsters (Mesocricetus auratus). Eur. J. Neurosci. 31, 16551663.

Haller, J., HalÃisz, J., Mikics, Ã., Kruk, M.R., 2004. Chronic glucocorticoid deficiency-induced abnormal aggression, autonomic hypoarousal, and social deficit in rats. J. Neuroendocrinol. 16, 550-557.

Haller, J., Makara, G.B., Barna, I., Kovacs, K., Nagy, J., Vecsernyes, M., 1996. Compression of the pituitary stalk elicits chronic increases in CSF vasopressin, oxytocin as well as in social investigation and aggressiveness. J. Neuroendocrinol. 8, 361-365.

Ho, H.P., Olsson, M., Westberg, L., Melke, J., Eriksson, E., 2001. The serotonin reuptake inhibitor fluoxetine reduces sex steroid-related aggression in female rats: an animal model of premenstrual irritability? Neuropsychopharmacology 24, 502-510.

Johns, J.M., Nelson, C.J., et al., 1998. Dose-dependent effects of multiple acute cocaine injections on maternal behavior and aggression in Sprague-Dawley rats. Dev. Neurosci. 20 (6) 525532.

Johnson, J.G., Cohen, P., Brown, J., Smailes, E.M., Bernstein, D.P., 1999. Childhood maltreatment increases risk for personality disorders during early adulthood. Arch. Gen. Psychiatr. 56, 600-606.

Kinsley, C., Svare, B., 1986. Prenatal stress reduces intermale aggression in mice. Physiol. Behav. 36, 783-786.

Kagerbauer, S.M., Martin, J., Schuster, T., Blobner, M., Kochs, E.F., Landgraf, R., 2013. Plasma oxytocin and vasopressin do not predict neuropeptide concentrations in the human cerebrospinal fluid. J. Neuroendocrinol. 11, 12038.

Koenen, K.C., 2005. Nature-nurture interplay: genetically informative designs contribute to understanding the effects of trauma and interpersonal violence. J. Interpers. Violence 20, 507-512.

Kruk, M.R., Van der Laan, C.E., Mos, J., Van der Poel, A.M., Meelis, W., Olivier, B., 1984. Comparison of aggressive behaviour induced by electrical stimulation in the hypothalamus of male and female rats. Prog. Brain Res. 61, 303-314.

Laporte, L., Paris, J., Guttman, H., Russell, J., 2011. Psychopathology, childhood trauma, and personality traits in patients with borderline personality disorder and their sisters. J. Pers. Disord. 25, 448-462.

Lewis, D.O., Yeager, C.A., Cobham-Portorreal, C.S., Klein, N., Showalter, C., Anthony, A., 1991. A follow-up of female delinquents: maternal contributions to the perpetuation of deviance. J. Am. Acad. Child Adolesc. Psychiatr. 30, 197-201.

Lovick, T.A., Griffiths, J.L., Dunn, S.M.J., Martin, I.L., 2005. Changes in GABAA receptor subunit expression in the midbrain during the oestrous cycle in Wistar rats. Neuroscience 131, 397-405.

Malick, J.B., 1979. The pharmacology of isolation-induced aggressive behavior in mice. Curr. Dev. Psychopharmacol. 5, 1-27.

Marquez, C., Poirier, G.L., Cordero, M.I., Larsen, M.H., Groner, A., Marquis, J., Magistretti, P.J., Trono, D., Sandi, C., 2013. Peripuberty stress leads to abnormal aggression, altered amygdala and orbitofrontal reactivity and increased prefrontal MAOA gene expression. Transl. Psychiatr. 15, 144.

More, L., 2008. Intra-female aggression in the mouse (Mus musculus domesticus) is linked to the estrous cycle regularity but not to ovulation. Aggress. Behav. 34, 46-50. 
Parmigiani, S., Brain, P.F., et al., 1988. Different patterns of biting attack employed by lactating female mice (Mus domesticus) in encounters with male and female conspecific intruders. J. Comp. Psychol. 102 (3) 287-293.

Paus, T., Keshavan, M., Giedd, J.N., 2008. Why do many psychiatric disorders emerge during adolescence? Nat. Rev. Neurosci. 9, 947-957.

Putkonen, H., Komulainen, E.J., Virkkunen, M., Eronen, M., Lönnqvist, J., 2003. Risk of repeat offending among violent female offenders with psychotic and personality disorders. Am. J. Psychiatr. 160, 947-951.

Sachser, N., 1993. The ability to arrange with conspecifics depends on social experiences around puberty. Physiol. Behav. 53, 539-544.

Seroczynski, A.D., Bergeman, C.S., Coccaro, E.F., 1999. Etiology of the impulsivity/aggression relationship: genes or environment? Psychiatr. Res. 86, 41-57.

Strathearn, L., Lyengar, U., Fonagy, P., Kim, S., 2012. Maternal oxytocin response during mother-infant interaction: associations with adult temperament. Horm. Behav. 61, 429-435.

Thompson, R.R., George, K., Walton, J.C., Orr, S.P., Benson, J., 2006. Sex-specific influences of vasopressin on human social communication. Proc. Natl. Acad. Sci. U. S. A. 103, 7889-7894.

Umukoro, S., Aladeokin, A.C., Eduviere, A.T., 2012. Aggressive behaviour: a comprehensive review of its neurochemical mechanisms and management. Aggress. Violent Behav. .

Veenema, A.H., Beiderbeck, D.I., Lukas, M., Neumann, I.D., 2010. Distinct correlations of vasopressin release within the lateral septum and the bed nucleus of the stria terminalis with the display of intermale aggression. Horm. Behav. 58, 273-281.

Veenema, A.H., Blume, A., Niederle, D., Buwalda, B., Neumann, I.D., 2006. Effects of early life stress on adult male aggression and hypothalamic vasopressin and serotonin. Eur. J. Neurosci. 24, $1711-1720$.

Vom Saal, F.S., Even, M.D., Quadagno, D.M., 1991. Effects of maternal stress on puberty, fertility and aggressive behavior of female mice from different intrauterine positions. Physiol. Behav. 49, 1073-1078.

Walsh, Z., Swogger, M.T., O’Connor, B.P., Chatav Schonbrun, Y., Shea, M.T., Stuart, G.L., 2010. Subtypes of partner violence perpetra tors among male and female psychiatric patients. J. Abnorm. Psychol. 119, 563-574.

Wolff, N., Shi, J., 2012. Childhood and adult trauma experiences of incarcerated persons and their relationship to adult behavioral health problems and treatment. Int. J. Environ. Res. Public Health 9, 1908-1926.

Wotjak, C.T., Ganster, J., Kohl, G., Holsboer, F., Landgraf, R., Engelmann, M., 1998. Dissociated central and peripheral release of vasopressin, but not oxytocin, in response to repeated swim stress: new insights into the secretory capacities of peptidergic neurons. Neuroscience 85, 1209-1222.

Zanarini, M.C., Frankenburg, F.R., Yong, L., Raviola, G., Bradford Reich, D., Hennen, J., Hudson, J.I., Gunderson, J.G., 2004. Borderline psychopathology in the first-degree relatives of borderline and axis II comparison probands. J. Pers. Disord. 18, 439-447. 